\title{
Multiplier operators on product spaces
}

\author{
by \\ Hung Viet Le (Weatherford, OK)
}

\begin{abstract}
The author proves the boundedness for a class of multiplier operators on product spaces. This extends a result obtained by Lung-Kee Chen in 1994.
\end{abstract}

1. Introduction. Fourier analysis and $H^{p}$-theory on product domains have recently been developed by many authors (for more detailed and complete treatment of these matters, the reader may consult e.g. S.-Y. Chang and R. Fefferman [2] and R. Fefferman [4]).

In 1987, Robert Fefferman gave a powerful theorem (see [4]), which effectively helps us study the boundedness of linear operators on the product spaces $H^{p}\left(\mathbb{R}^{n_{1}} \times \mathbb{R}^{n_{2}}\right)$. Thanks to this theorem, it suffices to check the boundedness of the linear operators acting on $H^{p}$ rectangle atoms, even though such atoms are not dense in the product domains (see counterexample of L. Carleson [1]). For convenience, we state Fefferman's theorem below:

Theorem 1 (see [4]). Let $T$ be a bounded linear operator on $L^{2}\left(\mathbb{R}^{n_{1}} \times\right.$ $\left.\mathbb{R}^{n_{2}}\right)$. Suppose that if $a$ is an $H^{p}\left(\mathbb{R}^{n_{1}} \times \mathbb{R}^{n_{2}}\right)$ rectangle atom $(0<p \leq 1)$ supported on $R$, then

$$
\int_{{ }^{c} R_{r}}|T(a)|^{p}\left(x_{1}, x_{2}\right) d x_{1} d x_{2} \leq C r^{-\sigma} \quad \text { for all } r \geq 2
$$

and some fixed $\sigma>0$, where ${ }^{\mathrm{c}} R_{r}$ denotes the complement of the $r$-fold enlargement of $R$. Then $T$ is a bounded operator from $H^{p}\left(\mathbb{R}^{n_{1}} \times \mathbb{R}^{n_{2}}\right)$ to $L^{p}\left(\mathbb{R}^{n_{1}} \times \mathbb{R}^{n_{2}}\right)$.

Based on Fefferman's Theorem and some ideas from [6], Lung-Kee Chen proved the following theorem.

Theorem 2 (see [3]). Let $k=\left\lfloor n_{1}(1 / p-1 / 2)\right\rfloor+1, l=\left\lfloor n_{2}(1 / p-1 / 2)\right\rfloor$ $+1,0<p \leq 1$. Suppose $m \in C^{k}\left(\mathbb{R}^{n_{1}}\right) \times C^{l}\left(\mathbb{R}^{n_{2}}\right)$ and

$$
\left|\partial_{\zeta}^{\alpha} \partial_{\eta}^{\beta} m(\zeta, \eta)\right| \leq C|\zeta|^{-|\alpha|}|\eta|^{-|\beta|} \quad \text { for }|\alpha| \leq k,|\beta| \leq l \text {. }
$$

2000 Mathematics Subject Classification: 42B15, 42B30. 
Then the multiplier operator $T_{m}$, defined on the Fourier transform side by $\widehat{T}_{m} f(\zeta, \eta)=m(\zeta, \eta) \widehat{f}(\zeta, \eta)$, maps $H^{q}\left(\mathbb{R}^{n_{1}} \times \mathbb{R}^{n_{2}}\right)$ boundedly to $L^{q}\left(\mathbb{R}^{n_{1}} \times \mathbb{R}^{n_{2}}\right)$ for $p \leq q \leq 2$.

Remarks. (a) R. Fefferman and K. C. Lin [5] have obtained the above result for the case $p=1$ under a weaker hypothesis:

$$
\int_{s_{1}<|\zeta|<2 s_{1}} \int_{s_{2}<|\eta|<2 s_{2}}\left|\partial_{\zeta}^{\alpha} \partial_{\eta}^{\beta} m(\zeta, \eta)\right|^{2} d \zeta d \eta \leq C s_{1}^{-2|\alpha|+n_{1}} s_{2}^{-2|\beta|+n_{2}} .
$$

(b) It is worth to mention the theorem below, obtained by A. Nilsson [8].

Theorem 3 (see [8]). Assume that $m \in C^{k}\left(\mathbb{R}^{n} \backslash\{0\}\right)$ and

$$
\int_{\Delta_{j}} \sum_{|\alpha| \leq k}\left|2^{j|\alpha|} \partial^{\alpha} m(\zeta)\right|^{2} d \zeta \leq 2^{n j(2-q) / q}, \quad j \in \mathbb{Z}
$$

where $k$ is the least integer $>n(2-q) /(2 q), 1 \leq q \leq 2$, and $\Delta_{j}=\{\zeta \in$ $\left.\mathbb{R}^{n}: 2^{j-1} \leq|\eta| \leq 2^{j+1}\right\}$. Then the convolution by $K=\widehat{m}$ maps $H^{1}\left(\mathbb{R}^{n}\right)$ to $L^{q}\left(\mathbb{R}^{n}\right)$.

The purpose of this note is to obtain the same result as in Theorem 2, but with a different hypothesis.

TheOREM 4. Let $m$ be a bounded function in $C^{k}\left(\mathbb{R}^{n_{1}}\right) \times C^{l}\left(\mathbb{R}^{n_{2}}\right)$, where

$$
k=\left\lfloor n_{1}(1 / p-1 / 2)\right\rfloor+1, \quad l=\left\lfloor n_{2}(1 / p-1 / 2)\right\rfloor+1, \quad 0<p \leq 1 .
$$

Suppose that

$$
\begin{aligned}
\int_{\Delta_{i} \times \Delta_{j}} \sum_{|\alpha| \leq k} \sum_{|\beta| \leq l}\left|2^{i|\alpha|} 2^{j|\beta|} \partial_{\zeta}^{\alpha} \partial_{\eta}^{\beta}\left(\zeta^{u} \eta^{v} m(\zeta, \eta)\right)\right|^{2} d \zeta d \eta & \\
& \leq C 2^{n_{1} i} 2^{n_{2} j} 2^{2 i|u|} 2^{2 j|v|}
\end{aligned}
$$

where $\Delta_{i}=\left\{\zeta \in \mathbb{R}^{n_{1}}: 2^{i} \leq|\zeta| \leq 2^{i+1}\right\}$, and a similar definition for $\Delta_{j}$,

$$
\sup _{\zeta \in \mathbb{R}^{n_{1}}}\left\{\int_{\Delta_{j}} \sum_{|\beta| \leq l}\left|2^{j|\beta|} \partial_{\eta}^{\beta}\left(\eta^{v} m(\zeta, \eta)\right)\right|^{2} d \eta\right\} \leq C 2^{n_{2} j} 2^{2 j|v|},
$$

and

$$
\sup _{\eta \in \mathbb{R}^{n_{2}}}\left\{\int_{\Delta_{i}} \sum_{|\alpha| \leq k}\left|2^{i|\alpha|} \partial_{\zeta}^{\alpha}\left(\zeta^{u} m(\zeta, \eta)\right)\right|^{2} d \zeta\right\} \leq C 2^{n_{1} i} 2^{2 i|u|} .
$$

Then the linear operator $T$ defined by $\widehat{T} f(\zeta, \eta)=m(\zeta, \eta) \widehat{f}(\zeta, \eta)$ maps $H^{q}\left(\mathbb{R}^{n_{1}} \times \mathbb{R}^{n_{2}}\right)$ boundedly to $L^{q}\left(\mathbb{R}^{n_{1}} \times \mathbb{R}^{n_{2}}\right)$ for $p \leq q \leq 2$.

Proof. Throughout the proof, $C$ denotes a constant which is not necessarily the same each time it appears. The proof is an application of Theorem 1 , and the idea of the proof is closely related to that of [3].

Let $a(x, y)$ denote an $H^{p}\left(\mathbb{R}^{n_{1}} \times \mathbb{R}^{n_{2}}\right)(0<p \leq 1)$ rectangle atom such that 
(a) $a(x, y)$ is supported on a rectangle $R=I \times J$, where $I$ and $J$ are cubes in $\mathbb{R}^{n_{1}}$ and $\mathbb{R}^{n_{2}}$ respectively,

(b) $\|a\|_{L^{2}} \leq R^{1 / 2-1 / p}=|I|^{1 / 2-1 / p}|J|^{1 / 2-1 / p}$,

(c) $\int_{I} x^{\alpha} a(x, y) d x=0$ for all $y \in J$ and $|\alpha| \leq k$, and

(d) $\int_{J} y^{\beta} a(x, y) d y=0$ for all $x \in I$ and $|\beta| \leq l$,

where $k$ and $l$ are defined in Theorem 4 .

By Theorem 1, it suffices to show that

$$
\int_{{ }^{c} R_{r}}|T(a)|^{p}\left(x_{1}, x_{2}\right) d x_{1} d x_{2} \leq C r^{\sigma} \quad \text { for all } r \geq 2,
$$

and some fixed $\sigma>0$.

Let $\phi$ be a smooth function on $\mathbb{R}$ such that $\widehat{\phi}(t)$ has compact support $\{t \in \mathbb{R}: 1 / 2 \leq|t| \leq 2\}$ and $\sum_{j \in \mathbb{Z}} \widehat{\phi}\left(2^{-j}|t|\right)=1$ for all $t \neq 0$.

Let $m_{i j}$ be defined by $m_{i j}(\zeta, \eta)=m(\zeta, \eta) \widehat{\phi}\left(2^{-i}|\zeta|\right) \widehat{\phi}\left(2^{-j}|\eta|\right)$ and let $\widehat{T}_{i j} f(\zeta, \eta)=m_{i j}(\zeta, \eta) \widehat{f}(\zeta, \eta) \equiv\left(K_{i j} * f\right)^{\wedge}(\zeta, \eta)$. It is clear that $T f=$ $\sum_{i, j} T_{i j} f$.

By Leibniz's formula and the hypothesis of Theorem 4, we have

$$
\begin{array}{rl}
\int \sum_{|\alpha| \leq k} \sum_{|\beta| \leq l}\left|2^{i|\alpha|} 2^{j|\beta|} \partial_{\zeta}^{\alpha} \partial_{\eta}^{\beta}\left(\zeta^{u} \eta^{v} m_{i j}(\zeta, \eta)\right)\right|^{2} & d \zeta d \eta \\
& \leq C 2^{n_{1} i} 2^{n_{2} j} 2^{2 i|u|} 2^{2 j|v|},
\end{array}
$$

$$
\begin{aligned}
& \sup _{\zeta \in \mathbb{R}^{n_{1}}}\left\{\int_{\mathbb{R}^{n_{2}}} \sum_{|\beta| \leq l}\left|2^{j|\beta|} \partial_{\eta}^{\beta}\left(\eta^{v} m_{j}(\zeta, \eta)\right)\right|^{2} d \eta\right\} \leq C 2^{n_{2} j} 2^{2 j|v|}, \\
& \sup _{\eta \in \mathbb{R}^{n_{2}}}\left\{\int_{\mathbb{R}^{n_{1}}} \sum_{|\alpha| \leq k}\left|2^{i|\alpha|} \partial_{\zeta}^{\alpha}\left(\zeta^{u} m_{i}(\zeta, \eta)\right)\right|^{2} d \zeta\right\} \leq C 2^{n_{1} i} 2^{2 i|u|},
\end{aligned}
$$

where $m_{j}(\zeta, \eta)=\sum_{i \in \mathbb{Z}} m_{i j}(\zeta, \eta)$ and $m_{i}(\zeta, \eta)=\sum_{j \in \mathbb{Z}} m_{i j}(\zeta, \eta)$.

We now partition ${ }^{\mathrm{c}} R_{r}$ into three regions:

$$
\begin{aligned}
& { }^{\mathrm{c}} R_{r}^{1}=\left\{(\zeta, \eta): \zeta \in{ }^{\mathrm{c}} I_{r}, \eta \in J_{2}\right\}, \\
& { }^{\mathrm{c}} R_{r}^{2}=\left\{(\zeta, \eta): \zeta \in I_{2}, \eta \in{ }^{\mathrm{c}} J_{r}\right\}, \quad{ }^{\mathrm{c}} R_{r}^{3}={ }^{\mathrm{c}} R_{r} \backslash\left({ }^{\mathrm{c}} R_{r}^{1} \cup{ }^{\mathrm{c}} R_{r}^{2}\right) .
\end{aligned}
$$

We may assume that the center of the rectangle $R=I \times J$ is at the origin $(0,0) \in \mathbb{R}^{n_{1}} \times \mathbb{R}^{n_{2}}$. We then write

$$
\begin{aligned}
T_{i j} a(x, y)= & \int_{I \times J} K_{i j}\left(x-x^{\prime}, y-y^{\prime}\right) a\left(x^{\prime}, y^{\prime}\right) d x^{\prime} d y^{\prime} \\
= & \int_{I \times J}\left\{K_{i j}\left(x-x^{\prime}, y-y^{\prime}\right)\right. \\
& \left.-\sum_{|\alpha| \leq \lambda_{1}-1} \frac{1}{\alpha !} \partial_{x}^{\alpha} K_{i j}\left(x, y-y^{\prime}\right)\left(-x^{\prime}\right)^{\alpha}\right\} a\left(x^{\prime}, y^{\prime}\right) d x^{\prime} d y^{\prime}
\end{aligned}
$$




$$
\begin{aligned}
= & \lambda_{1} \sum_{|\alpha|=\lambda_{1}} \frac{1}{\alpha !} \int_{I \times J} \int_{0}^{1}(1-t)^{\lambda_{1}-1} \partial_{x}^{\alpha} K_{i j}\left(x-t x^{\prime}, y-y^{\prime}\right) \\
& \times\left(-x^{\prime}\right)^{\alpha} a\left(x^{\prime}, y^{\prime}\right) d t d x^{\prime} d y^{\prime} \\
= & \lambda_{1} \sum_{|\alpha|=\lambda_{1}} \frac{1}{\alpha !} \int_{0}^{1} \int_{I \times J}(1-t)^{\lambda_{1}-1}\left\{\partial_{x}^{\alpha} K_{i j}\left(x-t x^{\prime}, y-y^{\prime}\right)\right. \\
& \left.-\sum_{|\beta| \leq \lambda_{2}-1} \frac{1}{\beta !} \partial_{y}^{\beta} \partial_{x}^{\alpha} K_{i j}\left(x-t x^{\prime}, y\right)\left(-y^{\prime}\right)^{\beta}\right\}\left(-x^{\prime}\right)^{\alpha} a\left(x^{\prime}, y^{\prime}\right) d y^{\prime} d x^{\prime} d t \\
= & \lambda_{1} \lambda_{2} \sum_{|\alpha|=\lambda_{1}} \frac{1}{\alpha !} \sum_{|\beta|=\lambda_{2}} \frac{1}{\beta !}\left\{\int_{I \times J} \int_{0}^{1} \int_{0}^{1}(1-t)^{\lambda_{1}-1}(1-s)^{\lambda_{2}-1}\right. \\
& \left.\times\left(-x^{\prime}\right)^{\alpha}\left(-y^{\prime}\right)^{\beta} \partial_{y}^{\beta} \partial_{x}^{\alpha} K_{i j}\left(x-t x^{\prime}, y-s y^{\prime}\right) a\left(x^{\prime}, y^{\prime}\right) d s d t d x^{\prime} d y^{\prime}\right\}
\end{aligned}
$$

where $0 \leq \lambda_{1} \leq k$ and $0 \leq \lambda_{2} \leq l$.

Notice that if $\lambda_{1}=0$ or $\lambda_{2}=0$, then we do not subtract the Taylor polynomials appearing in (4) above.

The integral on the right-hand side of (4) is dominated by

$$
\int_{I \times J} \int_{0}^{1} \int_{0}^{1}\left|\left(-x^{\prime}\right)^{\alpha}\left(-y^{\prime}\right)^{\beta} \partial_{y}^{\beta} \partial_{x}^{\alpha} K_{i j}\left(x-t x^{\prime}, y-s y^{\prime}\right) a\left(x^{\prime}, y^{\prime}\right)\right| d s d t d x^{\prime} d y^{\prime}
$$$$
\leq\|a\|_{L^{2}}\left\{\int_{I \times J} \int_{0}^{1} \int_{0}^{1}\left|\left(-x^{\prime}\right)^{\alpha}\left(-y^{\prime}\right)^{\beta} \partial_{y}^{\beta} \partial_{x}^{\alpha} K_{i j}\left(x-t x^{\prime}, y-s y^{\prime}\right)\right|^{2} d s d t d x^{\prime} d y^{\prime}\right\}^{1 / 2}
$$$$
\leq|I|^{1 / 2-1 / p+\lambda_{1} / n_{1}}|J|^{1 / 2-1 / p+\lambda_{2} / n_{2}}
$$

$$
\begin{aligned}
& \times\left\{\int_{I \times J} \int_{0}^{1} \int_{0}^{1}\left|\partial_{y}^{\beta} \partial_{x}^{\alpha} K_{i j}\left(x-t x^{\prime}, y-s y^{\prime}\right)\right|^{2} d s d t d x^{\prime} d y^{\prime}\right\}^{1 / 2} \\
& \equiv|I|^{1 / 2-1 / p+\lambda_{1} / n_{1}}|J|^{1 / 2-1 / p+\lambda_{2} / n_{2}} L_{i j}(x, y) .
\end{aligned}
$$

We now estimate $\int_{{ }^{c} R_{r}^{3}}\left|L_{i j}(x, y)\right|^{p} d x d y$. By Hölder's inequality, we have

$$
\begin{aligned}
& \int_{{ }^{\mathrm{c}} R_{r}^{3}}\left|L_{i j}(x, y)\right|^{p} d x d y \\
& \quad=\int_{{ }^{c} R_{r}^{3}}\left\{A(x)^{-k p / 2} B(y)^{-l p / 2}\right\}\left\{A(x)^{k / 2} B(y)^{l / 2} L_{i j}(x, y)\right\}^{p} d x d y
\end{aligned}
$$




$$
\begin{aligned}
\leq & \left\{\int_{{ }^{c} R_{r}^{3}} A(x)^{-k p /(2-p)} B(y)^{-l p /(2-p)}\right\}^{(2-p) / 2} \\
& \times\left\{\int_{{ }_{\mathrm{c}} R_{r}^{3}} A(x)^{k} B(y)^{l}\left|L_{i j}(x, y)\right|^{2} d x d y\right\}^{p / 2} .
\end{aligned}
$$

Here $A(x)=1+2^{2(i-1)}|x|^{2}$ and $B(y)=1+2^{2(j-1)}|y|^{2}$. Observe that

$$
\begin{aligned}
& \left\{\int_{{ }^{c} R_{r}^{3}} A(x)^{-k p /(2-p)} B(y)^{-l p /(2-p)} d x d y\right\}^{(2-p) / 2} \\
\leq & C 2^{-i k p} 2^{-j l p}|I|^{-k p / n_{1}+(2-p) / 2}|J|^{-l p / n_{2}+(2-p) / 2} r^{-k p-l p+\left(n_{1}+n_{2}\right)(2-p) / 2}
\end{aligned}
$$

Meanwhile,

$$
\begin{array}{rl}
\int_{{ }^{\mathrm{c}} R_{r}^{3}} A(x)^{k} & B(y)^{l}\left|L_{i j}(x, y)\right|^{2} d x d y \\
= & \int_{{ }^{\mathrm{c}} R_{r}^{3}}\left(1+2^{2(i-1)}|x|^{2}\right)^{k}\left(1+2^{2(j-1)}|y|^{2}\right)^{l} \\
& \times\left\{\int_{I \times J} \int_{0}^{1} \int_{0}^{1}\left|\partial_{y}^{\beta} \partial_{x}^{\alpha} K_{i j}\left(x-t x^{\prime}, y-s y^{\prime}\right)\right|^{2} d s d t d x^{\prime} d y^{\prime}\right\} d x d y .
\end{array}
$$

Since $\left(x^{\prime}, y^{\prime}\right) \in I \times J,(x, y) \in{ }^{\mathrm{c}} R_{r}^{3}$, and $0 \leq s, t \leq 1$, we have

$$
|x| \leq \frac{\left|x-t x^{\prime}\right|}{|x|-|I|^{1 / n_{1}} / 2} \cdot|x| \leq 2\left|x-t x^{\prime}\right| .
$$

Similarly, $|y| \leq 2\left|y-s y^{\prime}\right|$. Therefore, the above integral is dominated by

$$
\begin{aligned}
\int_{{ }^{\mathrm{c}} R_{r}^{3}}(1+ & \left.2^{2 i}\left|x-t x^{\prime}\right|^{2}\right)^{k}\left(1+2^{2 j}\left|y-s y^{\prime}\right|^{2}\right)^{l} \\
& \times\left\{\int_{I \times J} \int_{0}^{1} \int_{0}^{1}\left|\partial_{y}^{\beta} \partial_{x}^{\alpha} K_{i j}\left(x-t x^{\prime}, y-s y^{\prime}\right)\right|^{2} d s d t d x^{\prime} d y^{\prime}\right\} d x d y \\
\leq & \int_{0}^{1} \int_{0}^{1} \int_{I \times J \mathbb{R}^{n_{1}} \times \mathbb{R}^{n_{2}}}\left(1+2^{2 i}\left|x-t x^{\prime}\right|^{2}\right)^{k}\left(1+2^{2 j}\left|y-s y^{\prime}\right|^{2}\right)^{l} \\
& \times\left|\partial_{y}^{\beta} \partial_{x}^{\alpha} K_{i j}\left(x-t x^{\prime}, y-s y^{\prime}\right)\right|^{2} d x d y d x^{\prime} d y^{\prime} d s d t \\
= & \int_{0}^{1} \int_{0}^{1} \int_{I \times J \mathbb{R}^{n_{1}} \times \mathbb{R}^{n_{2}}}\left(1+2^{2 i}|x|^{2}\right)^{k}\left(1+2^{2 j}|y|^{2}\right)^{l} \\
& \times\left|\partial_{y}^{\beta} \partial_{x}^{\alpha} K_{i j}(x, y)\right|^{2} d x d y d x^{\prime} d y^{\prime} d s d t
\end{aligned}
$$




$$
\begin{aligned}
& \leq|I| \cdot|J| \int_{\mathbb{R}^{n_{1}} \times \mathbb{R}^{n_{2}}}\left(1+2^{2 i}|x|^{2}\right)^{k}\left(1+2^{2 j}|y|^{2}\right)^{l}\left|\partial_{y}^{\beta} \partial_{x}^{\alpha} K_{i j}(x, y)\right|^{2} d x d y \\
&=|I| \cdot|J| \int_{\mathbb{R}^{n_{1}} \times \mathbb{R}^{n_{2}}}\left(\sum_{|u| \leq k} \frac{k !}{(k-|u|) ! u !}\left|2^{i|u|} x^{u}\right|^{2}\right) \\
& \times\left(\sum_{|v| \leq l} \frac{l !}{(l-|v|) ! v !}\left|2^{j|v|} y^{v}\right|^{2}\right)\left|\partial_{y}^{\beta} \partial_{x}^{\alpha} K_{i j}(x, y)\right|^{2} d x d y \\
& \leq C|I| \cdot|J| \sum_{|u| \leq k} \sum_{|v| \leq l \mathbb{R}^{n_{1}} \times \mathbb{R}^{n_{2}}}\left|2^{i|u|} x^{u} 2^{j|v|} y^{v} \partial_{y}^{\beta} \partial_{x}^{\alpha} K_{i j}(x, y)\right|^{2} d x d y \\
&=C|I| \cdot|J| \sum_{|u| \leq k} \sum_{|v| \leq l \mathbb{R}^{n_{1}} \times \mathbb{R}^{n_{2}}}\left\{2^{i|u|} 2^{j|v|} \partial_{\zeta}^{u} \partial_{\eta}^{v}\left(\left(\partial_{\zeta}^{\alpha} \partial_{\eta}^{\beta} K_{i j}\right)^{\wedge}(\zeta, \eta)\right)\right\}^{2} d \zeta d \eta \\
&=C|I| \cdot|J| \int_{\mathbb{R}^{n_{1}} \times \mathbb{R}^{n_{2}}} \sum_{|u| \leq k} \sum_{|v| \leq l}\left|2^{i|u|} 2^{j|v|} \partial_{\zeta}^{u} \partial_{\eta}^{v}\left(\zeta^{\alpha} \eta^{\beta} m_{i j}(\zeta, \eta)\right)\right|^{2} d \zeta d \eta \\
& \leq C|I| \cdot|J| 2^{n_{1} i} 2^{n_{2} j} 2^{2 i|\alpha|} 2^{2 j|\beta|}=C|I| \cdot|J| 2^{n_{1} i} 2^{n_{2} j} 2^{2 i \lambda_{1}} 2^{2 j \lambda_{2}} \cdot
\end{aligned}
$$

Here the last inequality follows from (1), and the last equality from $|\alpha|=\lambda_{1}$ and $|\beta|=\lambda_{2}$ (see (4)). Combining inequalities (6)-(8) yields

$$
\begin{aligned}
\int_{{ }^{\mathrm{c}} R_{r}^{3}}\left|L_{i j}(x, y)\right|^{p} d x d y \leq & C|I|^{1-k p / n_{1}}|J|^{1-l p / n_{2}} 2^{i p\left(n_{1} / 2+\lambda_{1}-k\right)} \\
& \times 2^{j p\left(n_{2} / 2+\lambda_{2}-l\right)} r^{-k p-l p+\left(n_{1}+n_{2}\right)(2-p) / 2} .
\end{aligned}
$$

Consequently, if we combine (4)-(9), we have

$$
\begin{array}{rl}
\int_{{ }^{c} R_{r}^{3}}\left|T_{i j} a\right|^{p} & d x d y \\
\leq & C \int\left\{|I|^{1 / 2-1 / p+\lambda_{1} / n_{1}}|J|^{1 / 2-1 / p+\lambda_{2} / n_{2}}\left|L_{i j}(x, y)\right|\right\}^{p} d x d y \\
\leq & C|I|^{p / 2+\lambda_{1} p / n_{1}-k p / n_{1}}|J|^{p / 2+\lambda_{2} p / n_{2}-l p / n_{2}} \\
& \times 2^{-i p\left(k-n_{1} / 2-\lambda_{1}\right)} 2^{-j p\left(l-n_{2} / 2-\lambda_{2}\right)} r^{-k p-l p+\left(n_{1}+n_{2}\right)(2-p) / 2} .
\end{array}
$$

Now if we set $\lambda_{1}=0=\lambda_{2} ; \lambda_{1}=k$ and $\lambda_{2}=0 ; \lambda_{1}=0$ and $\lambda_{2}=l$; and $\lambda_{1}=k, \lambda_{2}=l$ in inequality (10), we obtain

$$
\begin{aligned}
& \int_{{ }^{c} R_{r}^{3}}\left|T_{i j} a\right|^{p} d x d y \leq C r^{-k p-l p+\left(n_{1}+n_{2}\right)((2-p) / 2)} \\
& \times \min \left\{|I|^{p / 2-k p / n_{1}}|J|^{p / 2-l p / n_{2}} 2^{-i p\left(k-n_{1} / 2\right)} 2^{-j p\left(l-n_{2} / 2\right)}\right. \\
&|I|^{p / 2}|J|^{p / 2-l p / n_{2}} 2^{i p n_{1} / 2} 2^{-j p\left(l-n_{2} / 2\right)} \\
&|I|^{p / 2-k p / n_{1}}|J|^{p / 2} 2^{-i p\left(k-n_{1} / 2\right)} 2^{j p n_{2} / 2} \\
&\left.|I|^{p / 2}|J|^{p / 2} 2^{i p n_{1} / 2} 2^{j p n_{2} / 2}\right\}
\end{aligned}
$$


We now consider $\int_{c_{R_{r}^{2}}^{2}}\left|T_{i j} a\right|^{p} d x d y$. Let $T_{j} a=\sum_{i} T_{i j} a$. Observe that

$$
\widehat{T}_{j} a(\zeta, \eta)=m(\zeta, \eta) \widehat{\phi}\left(2^{-j}|\eta|\right) \widehat{a}(\zeta, \eta) \equiv \widehat{K}_{j}(\zeta, \eta) \widehat{a}(\zeta, \eta) .
$$

We write

$$
\begin{aligned}
& \int_{{ }^{c} R_{r}^{2}}\left|\sum_{i} T_{i j} a\right|^{p} d x d y=\int_{{ }^{c} R_{r}^{2}}\left|T_{j} a\right|^{p} d x d y \\
& \leq \int_{{ }^{c} R_{r}^{2}} B(y)^{-l p / 2}\left[B(y)^{l / 2}\left|T_{j} a\right|\right]^{p} d x d y \\
& \leq\left\{\int_{c R_{r}^{2}} B(y)^{-l p /(2-p)} d x d y\right\}^{(2-p) / 2}\left\{\int_{c R_{r}^{2}} B(y)^{l}\left|T_{j} a\right|^{2} d x d y\right\}^{p / 2},
\end{aligned}
$$

where again $B(y)=1+2^{2(j-1)}|y|^{2}$. It is clear that

$$
\begin{aligned}
\left\{\int_{{ }^{c} R_{r}^{2}} B(y)^{-l p /(2-p)}\right. & d x d y\}^{(2-p) / 2} \\
& \leq C|I|^{(2-p) / 2}|J|^{-l p / n_{2}+(2-p) / 2} 2^{-j l p} r^{n_{2}(2-p) / 2-l p}
\end{aligned}
$$

For the estimate of $\int_{c_{R_{r}^{2}}} B(y)^{l}\left|T_{j} a\right|^{2} d x d y$, we write

$$
\begin{aligned}
& T_{j} a(x, y)=K_{j} * a(x, y)=\int_{I \times J} K_{j}\left(x-x^{\prime}, y-y^{\prime}\right) a\left(x^{\prime}, y^{\prime}\right) d x^{\prime} d y^{\prime} \\
& =\int_{I \times J}\left\{K_{j}\left(x-x^{\prime}, y-y^{\prime}\right)\right. \\
& \left.\quad-\sum_{|\beta| \leq \lambda_{2}-1} \frac{1}{\beta !} \partial_{y}^{\beta} K_{j}\left(x-x^{\prime}, y\right)\left(-y^{\prime}\right)^{\beta}\right\} a\left(x^{\prime}, y^{\prime}\right) d x^{\prime} d y^{\prime} \\
& =\lambda_{2} \sum_{|\beta|=\lambda_{2}} \frac{1}{\beta !} \int_{I \times J} \int_{0}^{1}(1-s)^{\lambda_{2}-1} \partial_{y}^{\beta} K_{j}\left(x-x^{\prime}, y-s y^{\prime}\right)\left(-y^{\prime}\right)^{\beta} a\left(x^{\prime}, y^{\prime}\right) d s d x^{\prime} d y^{\prime} .
\end{aligned}
$$

Thus

$$
\begin{aligned}
\int_{{ }^{c} R_{r}^{2}} B(y)^{l}\left|T_{j} a\right|^{2} d x d y=\int_{{ }^{c} R_{r}^{2}} B(y)^{l} \mid \lambda_{2} \sum_{|\beta|=\lambda_{2}} \frac{1}{\beta !} \int_{0}^{1} \int_{I \times J}(1-s)^{\lambda_{2}-1} \\
\times\left.\partial_{y}^{\beta} K_{j}\left(x-x^{\prime}, y-s y^{\prime}\right)\left(-y^{\prime}\right)^{\beta} a\left(x^{\prime}, y^{\prime}\right) d x^{\prime} d y^{\prime} d s\right|^{2} d x d y
\end{aligned}
$$




$$
\begin{aligned}
\leq & C \sum_{|\beta|=\lambda_{2}} \int_{c} B(y)^{l} \\
& \times\left|\int_{0}^{1} \int_{I \times J}\left(-y^{\prime}\right)^{\beta} \partial_{y}^{\beta} K_{j}\left(x-x^{\prime}, y-s y^{\prime}\right) a\left(x^{\prime}, y^{\prime}\right) d x^{\prime} d y^{\prime} d s\right|^{2} d x d y \\
\leq & C \sum_{|\beta|=\lambda_{2}}\left\{\int _ { | y | \geq r | J | ^ { 1 / n _ { 2 } / 2 } } B ( y ) ^ { l } \left[\int_{0}^{1} \int_{J}\left|\left(-y^{\prime}\right)^{\beta}\right|\right.\right. \\
& \left.\left.\left.\times \int_{|x| \leq|I|^{1 / n_{1} / 2}}\left|\int_{I} \partial_{y}^{\beta} K_{j}\left(x-x^{\prime}, y-s y^{\prime}\right) a\left(x^{\prime}, y^{\prime}\right) d x^{\prime}\right|^{2} d x\right)^{1 / 2} d y^{\prime} d s\right]^{2} d y\right\}
\end{aligned}
$$

(by Minkowski's inequality for integrals)

$$
\begin{aligned}
\leq & C \sum_{|\beta|=\lambda_{2}}\left\{\int _ { | y | \geq r | J | ^ { 1 / n _ { 2 } / 2 } } B ( y ) ^ { l } \left[\int_{0}^{1} \int_{J}\left|\left(-y^{\prime}\right)^{\beta}\right|\right.\right. \\
& \left.\left.\times\left(\int_{\mathbb{R}^{n_{1}}}\left|\partial_{y}^{\beta K_{j}^{(1)}}\left(\zeta, y-s y^{\prime}\right) \widehat{a}^{(1)}\left(\zeta, y^{\prime}\right)\right|^{2} d \zeta\right)^{1 / 2} d y^{\prime} d s\right]^{2} d y\right\}
\end{aligned}
$$

(here $\widehat{a}^{(1)}$ means the Fourier Transform of $a$ with respect to the first variable, and a similar definition for $\left.\partial_{y}^{\beta} K_{j}^{(1)}\right)$

$$
\begin{aligned}
& \leq C|J|^{2 \lambda_{2} / n_{2}} \sum_{|\beta|=\lambda_{2}}\left\{\int _ { | y | \geq r | J | ^ { 1 / n _ { 2 } / 2 } } B ( y ) ^ { l } \left[\int _ { 0 } ^ { 1 } \int _ { J } \left(\int_{\mathbb{R}^{n_{1}}} \mid \partial_{y}^{\widehat{\beta} K_{j}^{(1)}}\left(\zeta, y-s y^{\prime}\right)\right.\right.\right. \\
& \left.\left.\left.\times\left.\widehat{a}^{(1)}\left(\zeta, y^{\prime}\right)\right|^{2} d \zeta\right)^{1 / 2} d y^{\prime} d s\right]^{2} d y\right\} \\
& \leq C|J|^{2 \lambda_{2} / n_{2}+1} \sum_{|\beta|=\lambda_{2}} \int_{|y| \geq r|J|^{1 / n_{2} / 2}} B(y)^{l} \int_{0}^{1} \int_{J} \int_{\mathbb{R}^{n_{1}}} \mid \partial_{y}^{\widehat{\beta} K_{j}^{(1)}}\left(\zeta, y-s y^{\prime}\right) \\
& \times\left.\widehat{a}^{(1)}\left(\zeta, y^{\prime}\right)\right|^{2} d \zeta d y^{\prime} d s d y \\
& =C|J|^{2 \lambda_{2} / n_{2}+1} \sum_{|\beta|=\lambda_{2}} \int_{|y| \geq r|J|^{1 / n_{2}} / 2} \int_{0}^{1} \int_{J} \int_{\mathbb{R}^{n_{1}}} \mid B(y)^{l / 2} \partial_{y}^{\widehat{\beta} K_{j}^{(1)}}\left(\zeta, y-s y^{\prime}\right) \\
& \times\left.\widehat{a}^{(1)}\left(\zeta, y^{\prime}\right)\right|^{2} d \zeta d y^{\prime} d s d y \\
& \leq C|J|^{2 \lambda_{2} / n_{2}+1} \sum_{|\beta|=\lambda_{2}} \int_{|y| \geq r|J|^{1 / n_{2} / 2}} \int_{0}^{1} \int_{J} \int_{\mathbb{R}^{n_{1}}} \mid\left(1+2^{2 j}\left|y-s y^{\prime}\right|^{2}\right)^{l / 2} \\
& \times\left.\partial_{y}^{\beta K_{j}^{(1)}}\left(\zeta, y-s y^{\prime}\right) \widehat{a}^{(1)}\left(\zeta, y^{\prime}\right)\right|^{2} d \zeta d y^{\prime} d s d y
\end{aligned}
$$




$$
\begin{aligned}
\leq C|J|^{2 \lambda_{2} / n_{2}+1} \sum_{|\beta|=\lambda_{2}} \int_{J} \int_{\mathbb{R}^{n_{1}}} \int_{\mathbb{R}^{n_{2}}} \mid\left(1+2^{2 j}|y|^{2}\right)^{l} \partial_{y}^{\widehat{\beta} K_{j}^{(1)}}(\zeta, y) & \\
& \times\left.\widehat{a}^{(1)}\left(\zeta, y^{\prime}\right)\right|^{2} d y d \zeta d y^{\prime} .
\end{aligned}
$$

But

$$
\begin{aligned}
\int_{\mathbb{R}^{n_{2}}}\left|\left(1+2^{2 j}|y|^{2}\right)^{l} \partial_{y}^{\widehat{\beta} K_{j}^{(1)}}(\zeta, y)\right|^{2} d y & \\
& \leq C \int_{\mathbb{R}^{n_{2}}} \sum_{|v| \leq l}\left|2^{j|v|} y^{v} \partial_{y}^{\beta} \widehat{K_{j}^{(1)}}(\zeta, y)\right|^{2} d y \\
& =C \int_{\mathbb{R}^{n_{2}}} \sum_{|v| \leq l}\left|2^{j|v|} \partial_{\eta}^{v}\left(\eta^{\beta} \widehat{K}_{j}(\zeta, \eta)\right)\right|^{2} d \eta \\
& =C \int_{\mathbb{R}^{n_{2}}} \sum_{|v| \leq l}\left|2^{j|v|} \partial_{\eta}^{v}\left(\eta^{\beta} m_{j}(\zeta, \eta)\right)\right|^{2} d \eta
\end{aligned}
$$

Combining (14) and (15), we have

$$
\begin{aligned}
& \int_{c_{r}^{2}} B(y)^{l}\left|T_{j} a\right|^{2} d x d y \\
& \leq C|J|^{2 \lambda_{2} / n_{2}+1} \sum_{|\beta|=\lambda_{2}} \int_{J} \int_{\mathbb{R}^{n_{1}}} \int_{\mathbb{R}^{n_{2}}} \sum_{|v| \leq l}\left|2^{j|v|} \partial_{\eta}^{v}\left(\eta^{\beta} m_{j}(\zeta, \eta)\right)\right|^{2} d \eta \\
& \quad \times\left|\widehat{a}^{(1)}\left(\zeta, y^{\prime}\right)\right|^{2} d \zeta d y^{\prime} \\
& \leq\left. C|J|^{2 \lambda_{2} / n_{2}+1} \sum_{|\beta|=\lambda_{2}} 2^{n_{2} j} 2^{2 j|\beta|} \int_{J}\left|\widehat{a}_{\mathbb{R}^{n_{1}}}\right| \hat{a}^{(1)}\left(\zeta, y^{\prime}\right)\right|^{2} d \zeta d y^{\prime} \\
& \leq C|J|^{2 \lambda_{2} / n_{2}+1} 2^{n_{2} j} 2^{2 j \lambda_{2}}\|a\|_{L^{2}}^{2} .
\end{aligned}
$$

The next to last inequality follows from (2). So

$$
\left\{\int_{c R_{r}^{2}} B(y)^{l}\left|T_{j} a\right|^{2} d x d y\right\}^{p / 2} \leq C|J|^{\lambda_{2} p / n_{2}+p / 2} 2^{j n_{2} p / 2} 2^{j \lambda_{2} p}\|a\|_{L^{2}}^{p} .
$$

Combining (12), (13), and (16) yields

$$
\begin{aligned}
& \int_{{ }^{\mathrm{c}} R_{r}^{2}}\left|T_{j} a\right|^{p} d x d y \\
& \leq C|J|^{-l p / n_{2}+p / 2+\lambda_{2} p / n_{2}} 2^{-j p\left(l-n_{2} / 2+\lambda_{2}\right)} r^{n_{2}((2-p) / 2)-l p} .
\end{aligned}
$$

If we set $\lambda_{2}=0$ and $\lambda_{2}=l$ in (17), we have

$$
\begin{aligned}
& \int_{{ }^{\mathrm{c}} R_{r}^{2}}\left|T_{j} a\right|^{p} d x d y \\
& \quad \leq C r^{n_{2}((2-p) / 2)-l p} \min \left\{|J|^{p / 2-l p / n_{2}} 2^{-j p\left(l-n_{2} / 2\right)},|J|^{p / 2} 2^{j p n_{2} / 2}\right\} .
\end{aligned}
$$


Observe that if we let $T_{i} a=\sum_{j} T_{i j} a$, where

$$
\widehat{T}_{i} a(\zeta, \eta)=m(\zeta, \eta) \widehat{\phi}\left(2^{-i}|\zeta|\right) \widehat{a}(\zeta, \eta) \equiv \widehat{K}_{i}(\zeta, \eta) \widehat{a}(\zeta, \eta)
$$

and write

$$
\int_{{ }^{c} R_{r}^{1}}\left|\sum_{j} T_{i j} a\right|^{p} d x d y=\int_{{ }^{c} R_{r}^{1}}\left|T_{i} a\right|^{p} d x d y,
$$

then by symmetry, we obtain

$$
\begin{aligned}
& \int_{{ }^{\mathrm{c}} R_{r}^{1}}\left|T_{i} a\right|^{p} d x d y \\
& \quad \leq C r^{n_{1}((2-p) / 2)-k p} \min \left\{|I|^{p / 2-k p / n_{1}} 2^{-i p\left(k-n_{1} / 2\right)},|I|^{p / 2} 2^{i p n_{1} / 2}\right\} .
\end{aligned}
$$

Finally, there exist $i_{0}, j_{0} \in \mathbb{Z}$ such that $2^{i_{0}-1} \leq|I|^{1 / n_{1}} \leq 2^{i_{0}}$ and $2^{j_{0}-1} \leq$ $|J|^{1 / n_{2}} \leq 2^{j_{0}}$. Because $k=\left\lfloor n_{1}(1 / p-1 / 2)\right\rfloor+1$ and $l=\left\lfloor n_{2}(1 / p-1 / 2)\right\rfloor+1$, there is a $\sigma>0$ such that

$$
\sigma \leq \min \left\{k p-n_{1}(2-p) / 2, l p-n_{2}(2-p) / 2\right\} .
$$

Thus inequalities (11), (18), and (19) respectively become

$$
\begin{gathered}
\text { (20) } \quad \int_{\mathrm{c} R_{r}^{3}}\left|T_{i j} a\right|^{p} d x d y \leq C r^{-2 \sigma} \min \left\{2^{-\left(i+i_{0}\right)\left(k p-n_{1} p / 2\right)} 2^{-\left(j+j_{0}\right)\left(l p-n_{2} p / 2\right)},\right. \\
2^{\left(i+i_{0}\right) n_{1} p / 2} 2^{-\left(j+j_{0}\right)\left(l p-n_{2} p / 2\right)}, 2^{-\left(i+i_{0}\right)\left(k p-n_{1} p / 2\right)} 2^{\left(j+j_{0}\right) n_{2} p / 2}, \\
\left.(21) \quad 2^{\left(i+i_{0}\right) n_{1} p / 2} 2^{\left(j+j_{0}\right) n_{2} p / 2}\right\} \\
\quad \int^{\mathrm{c} R_{r}^{2}}\left|T_{j} a\right|^{p} d x d y \leq C r^{-\sigma} \min \left\{2^{-\left(j+j_{0}\right)\left(l p-n_{2} p / 2\right)}, 2^{\left(j+j_{0}\right) n_{2} p / 2}\right\}, \\
\quad \int_{{ }^{c} R_{r}^{1}}\left|T_{i} a\right|^{p} d x d y \leq C r^{-\sigma} \min \left\{2^{-\left(i+i_{0}\right)\left(k p-n_{1} p / 2\right)}, 2^{\left(i+i_{0}\right) n_{1} p / 2}\right\} .
\end{gathered}
$$

Consequently, for $0<p \leq 1$ we have

$$
\begin{aligned}
\int_{{ }^{c} R_{r}}|T a|^{p} d x d y= & \int_{{ }^{c} R_{r}^{3}}|T a|^{p} d x d y+\int_{{ }^{c} R_{r}^{2}}|T a|^{p} d x d y+\int_{{ }^{c} R_{r}^{1}}|T a|^{p} d x d y \\
\leq & \sum_{i j} \int_{{ }^{c} R_{r}^{3}}\left|T_{i j} a\right|^{p} d x d y+\sum_{j} \int_{{ }^{c} R_{r}^{2}}\left|T_{j} a\right|^{p} d x d y \\
& +\sum_{i} \int_{{ }^{c} R_{r}^{1}}\left|T_{i} a\right|^{p} d x d y \\
\leq & C r^{-\sigma},
\end{aligned}
$$

where the last inequality follows from $(20)-(22)$. The proof is finished. 


\section{References}

[1] L. Carleson, A counterexample for measures bounded for $H^{p}$ for the bi-disc, Mittag Leffler Report No. 7, 1974.

[2] S.-Y. Chang and R. Fefferman, Some recent developments in Fourier analysis and $H^{p}$ theory on product domains, Bull. Amer. Math. Soc. 12 (1985), 1-43.

[3] L. K. Chen, The multiplier operator on the product spaces, Illinois J. Math. 38 (1994), 420-433.

[4] R. Fefferman, Harmonic analysis on product spaces, Ann. of Math. 126 (1987), 109130 .

[5] R. Fefferman and K. C. Lin, A sharp result on multiplier operators, preprint.

[6] G. Folland, Real Analysis: Modern Techniques and Their Applications, Wiley, 1984.

[7] A. Miyachi, On some Fourier multipliers for $H^{p}\left(\mathbb{R}^{n}\right)$, J. Fac. Sci. Univ. Tokyo Sect. 1A Math. 27 (1980), 157-179.

[8] A. Nilsson, Multipliers from $H^{1}$ to $L^{p}$, Ark. Mat. 36 (1998), 379-383.

Department of Mathematics

Southwestern Oklahoma State University

Weatherford, OK 73096, U.S.A.

E-mail: leh@swosu.edu 